\title{
Correlações genotípicas e análise de trilha em famílias de meios-irmãos de couve de folhas
}

\author{
Alcinei Mistico Azevedo(1), Rogério Seus ${ }^{(1)}$, Cristiane Lourenço Gomes ${ }^{(1)}$, Elis Marina de Freitas ${ }^{(1)}$, \\ Diego Mattos Candido(1), Derly José Henriques da Silva(1) e Pedro Crescêncio Souza Carneiro ${ }^{(1)}$
}

\begin{abstract}
(1)Universidade Federal de Viçosa, Campus Universitário, Departamento de Fitotecnia, Avenida Peter Henrry Rolfs, CEP $36570-000$ Viçosa, MG, Brasil. E-mail: alcineimistico@hotmail.com, rseusagro@hotmail.com, cristiane.lourenco@ufv.br, elis_stavanato@hotmail.com, diego_mc7@hotmail.com, derlyufv@gmail.com, carneiro@ufv.br
\end{abstract}

Resumo - O objetivo deste trabalho foi avaliar, por meio de correlação genotípica e análise de trilha, as associações entre características em famílias de meios-irmãos de couve e os possíveis efeitos da seleção truncada. Foram avaliadas 13 características em 24 famílias de meios-irmãos de couve. Realizaram-se quatro análises de trilha, tendo-se considerado as seguintes variáveis básicas: altura de planta, número de brotações, número de folhas por planta e diâmetro do caule. Cada análise considerou 12 características como variáveis explicativas. Constataram-se as maiores estimativas de correlação entre as seguintes características: comprimento e largura do pecíolo; número de folhas; massa de matéria fresca de folhas; e comprimento e largura de folhas. A seleção quanto ao menor número de brotações ocasiona a redução do comprimento do pecíolo; quanto ao maior número de folhas, proporciona aumento da produção de massa de matéria fresca de folhas e redução da massa de matéria fresca por folha; e, quanto ao maior diâmetro do caule, ocasiona o aumento da produção de massa de folhas frescas. A seleção truncada quanto à altura de plantas não ocasiona alterações expressivas às demais características avaliadas.

Termos para indexação: Brassica oleracea var. acephala, correlação genotípica, multicolinearidade, regressão em crista, seleção truncada.

\section{Genotypic correlation and path analysis of kale half-sib progenies}

\begin{abstract}
The objective of this work was to evaluate the associations between traits in half-sib families of kale, through genotypic correlation and path analysis, and possible effects of truncated selection. Thirteen traits were evaluated in 24 half-sib families of kale. Four path analysis were carried out, considering the following basic variables: plant height, number of shoots, number of leaves per plant, and stem diameter. Each analysis included 12 traits as explicative variables. The highest estimates of correlation were found between the following traits: length and width of petioles; number of leaves; leaf fresh weight; and length and width of the leaves. The selection for the smallest number of shoots leads to a reduction of petiole length; for the highest number of leaves, selection provides the increase of leaf fresh weight and the reduction of fresh weight per leaf; and, for the largest stem diameter, selection causes the production increase of fresh leaf weight. The truncated selection for plant height does not cause significant changes in the other evaluated traits.
\end{abstract}

Index terms: Brassica oleracea. var. acephala, genotypic correlation, multicollinearity, ridge path analysis, truncated selection.

\section{Introdução}

A couve - Brassica oleracea L. var. acephala DC. -, uma das formas mais antigas da família das brassicáceas, originou-se no leste do Mediterrâneo e tem sido utilizada como cultura alimentar desde 2000 anos A.C. (Balkaya \& Yanmaz, 2005). As brassicáceas vêm sendo selecionadas pelo homem desde então, conscientemente ou não, contudo, muito ainda deve ser feito para o melhoramento genético dessas culturas (Azevedo et al., 2012). Seu consumo tem aumentado, em virtude das novas formas de sua utilização na culinária e das recentes descobertas quanto às suas propriedades nutracêuticas (Naik \& Gupta, 2010; Novo et al., 2010; Azevedo et al., 2014). Sua ingestão reduz o risco de doenças crônicas relacionadas à idade, como problemas cardiovasculares e vários tipos de câncer (Moreno et al., 2006; Vilar et al., 2008; Soengas et al., 2011). 
O objetivo de qualquer programa de melhoramento é a obtenção de cultivares que superem com vantagens as pré-existentes. Ter vantagens adicionais só é possível se a nova variedade reunir simultaneamente uma série de fenótipos favoráveis quanto aos caracteres de interesse. No melhoramento genético da couve, tem-se procurado selecionar genótipos com menor altura, menor número de brotações, maior número de folhas por planta e maior diâmetro do caule. Todas estas características poderão possibilitar maior adensamento, maior rendimento por área e redução de tratos culturais, como a desbrota e o tutoramento, o que permite a maior rentabilidade da cultura (Azevedo et al., 2012, 2014).

Porém, a seleção truncada pode trazer consequências indesejáveis quanto às demais características de interesse agronômico, pois, pode haver associação entre características como consequência de pleiotropia ou ligação dos genes (Entringer et al., 2014). Assim, para o melhoramento genético da couve, é necessário conhecer a associação entre as características, para que sejam identificadas os possíveis efeitos da seleção quanto à altura de plantas, ao número de brotações, ao número de folhas por planta e ao diâmetro do caule -, sobre as demais características de interesse agronômico.

As associações existentes entre os caracteres são, em geral, avaliadas por meio das correlações fenotípicas, genotípicas e ambientais (Cruz et al., 2012). A correlação fenotípica tem causas genéticas e ambientais, mas somente as genéticas envolvem associação de natureza herdável que pode ser utilizada para orientar programas de melhoramento. Porém, a correlação estimada pode não representar a verdadeira associação entre dois caracteres, uma vez que uma alta ou baixa associação pode ocorrer por influência de um terceiro caráter ou conjunto de caracteres (Toebe \& Cargnelutti Filho, 2013).

Neste sentido, o método estatístico da análise de trilha permite estudar mais detalhadamente as relações entre as características por meio da estimação de coeficientes que caracterizam a influência que um caráter exerce sobre o outro. Logo, a associação entre os caracteres pode ser desdobrada em efeitos diretos e indiretos por um sistema causal que explica as inter-relações entre as variáveis (Teixeira et al., 2012).

O objetivo deste trabalho foi avaliar, por meio da correlação genotípica e da análise de trilha, as associações entre características em famílias de meios-irmãos de couve e os possíveis efeitos da seleção truncada.

\section{Material e Métodos}

O experimento foi conduzido de 26/1/2013 a 9/11/2013, na Horta Velha - horta de pesquisa - da Universidade Federal de Viçosa (UFV), Viçosa, MG (204' $14 " \mathrm{~S}, 42^{\circ} 52^{\prime} 53^{\prime \prime} \mathrm{W}$, a $648,74 \mathrm{~m}$ de altitude). Segundo a classificação de Köppen, o clima regional é do tipo Cwa, com $80 \%$ de média anual de umidade relativa do ar, 26,4 e $14,8^{\circ} \mathrm{C}$ de médias anuais de temperaturas máxima e mínima, respectivamente, e $1.221,4 \mathrm{~mm}$ de precipitação pluvial anual.

Vinte e quatro famílias de meios-irmãos de couve, provenientes de clones do banco de germoplasma da Universidade Federal dos Vales do Jequitinhonha e Mucuri (UFVJM), foram avaliadas em delineamento de blocos ao acaso, com quatro repetições e cinco plantas por parcela. A semeadura foi realizada no dia 26/01/2013, em bandejas de poliestireno expandido, com 128 células, em substrato comercial Plantmax Hortaliças, acondicionadas em ambiente protegido sob sombrite, com $50 \%$ de sombreamento, e submetidas a irrigações diárias. No dia $13 / 3 / 2013$, as mudas foram transplantadas para canteiros com largura aproximada de 2,50 m e 0,30 m de altura, com espaçamento de $1,00 \times 0,50 \mathrm{~m}$.

A partir do dia 13/4/2013 até o dia 9/11/2013, realizaram-se colheitas de 14 em 14 dias, o que totalizou 15 avaliações. Em cada avaliação, foram consideradas cinco plantas por parcela, em que foram mensurados os seguintes caracteres: número de brotações (quando estas foram removidas), número de folhas comercializáveis, massa de matéria fresca de folhas comercializáveis e massa de matéria fresca por folha. Foram consideradas como comercializáveis as folhas expandidas com comprimento do limbo foliar maior do que $15 \mathrm{~cm}$ e sem sinais de senescência (Azevedo et al., 2012).

No dia 6/7/2013, avaliou-se a altura das plantas, tendo-se medido a planta do nível do solo até a extremidade da folha mais alta, com o auxílio de uma fita métrica graduada em centímetros, e o diâmetro do caule, que foi medido com paquímetro na metade da altura da planta.

Na quinta folha expandida mais nova de cada planta, avaliaram-se os seguintes parâmetros: o comprimento e a largura do limbo foliar, medidos com régua graduada em centímetros; a razão entre o comprimento e a largura do limbo foliar; o diâmetro do centro do pecíolo; a espessura da base do pecíolo, medida com paquímetro, em milímetros; o comprimento do pecíolo, 
medido com régua graduada em centímetros, a partir de sua inserção no caule até o início do limbo foliar; e as razões entre o comprimento da folha e o comprimento do pecíolo. A quinta folha expandida mais nova foi escolhida para fins de padronização na avaliação, pois esta folha sempre apresenta grau de desenvolvimento similar na maioria dos genótipos.

Como as características número de folhas comercializáveis, massa de matéria fresca de folhas, massa de matéria fresca por folha e número de brotações foram avaliadas em várias colheitas (medidas repetidas), utilizou-se o modelo estatístico $\mathrm{y}=\mathrm{Xm}+\mathrm{Za}+\mathrm{Wp}+\mathrm{Ts}+\varepsilon$, em que: y é o vetor de dados; $\mathrm{y} \sim \mathrm{N}(\mathrm{Xm}, \mathrm{V}), \mathrm{V}=\mathrm{ZA} \sigma_{\mathrm{a}}^{2} \mathrm{Z}^{\prime}+\mathrm{WI} \sigma_{\mathrm{p}}^{2} \mathrm{~W}^{\prime}+\mathrm{TI} \sigma_{\mathrm{s}}^{2} \mathrm{~T}^{\prime}+\mathrm{I} \sigma_{\varepsilon}^{2}$; A é a matriz de parentesco entre os indivíduos; $\sigma_{a}^{2}$ é a variância genética aditiva; I é uma matriz identidade; $\sigma_{\mathrm{p}}^{2}$ é a variância ambiental entre parcelas; $\sigma_{\mathrm{s}}^{2}$ é a variância dos efeitos permanentes; $\sigma_{\varepsilon}^{2}$ é a variância residual; $m$ é o vetor dos efeitos das combinações medição-repetição, considerados como fixos, somados à média geral; a é o vetor dos efeitos genéticos aditivos individuais, considerados como aleatórios, em que $\mathrm{a} \sim \mathrm{N}\left(0, A \sigma_{\mathrm{a}}^{2}\right)$; $\mathrm{p}$ é o vetor dos efeitos de parcela (aleatórios), em que $\mathrm{p} \sim \mathrm{N}\left(0, \mathrm{I} \sigma_{\mathrm{p}}^{2}\right)$; s é o vetor dos efeitos permanentes (aleatórios), em que $\mathrm{s} \sim \mathrm{N}\left(0, \mathrm{I} \sigma_{\mathrm{s}}^{2}\right)$; e $\varepsilon$ é o vetor de erros (aleatórios), em que e $\sim \mathrm{N}\left(0, \mathrm{I} \sigma_{\varepsilon}^{2}\right)$. As letras maiúsculas representam as matrizes de incidência para os referidos efeitos.

Para as características altura de plantas, diâmetro do caule, comprimento e largura do limbo foliar, razão entre o comprimento e largura do limbo foliar, diâmetro do centro do pecíolo, espessura da base do pecíolo, comprimento do pecíolo e razão entre o comprimento da folha e o comprimento do pecíolo, mensuradas em uma única avaliação por planta, utilizou-se o modelo $\mathrm{y}=\mathrm{Xr}+\mathrm{Za}+\mathrm{Wp}+\varepsilon$, em que: y é o vetor de dados, em que $\mathrm{y} \sim \mathrm{N}(\mathrm{Xr}, \mathrm{V}), \mathrm{V}=\mathrm{ZA} \sigma_{\mathrm{a}}^{2} \mathrm{Z}^{\prime}+\mathrm{WI} \sigma_{\mathrm{c}}^{2} \mathrm{~W}^{\prime}+\mathrm{I} \sigma_{\varepsilon}^{2}$; é a variância genética aditiva; $\sigma_{\mathrm{c}}^{2}$ é a variância ambiental entre parcelas; $\sigma_{\varepsilon}^{2}$ é a variância residual; $r$ é o vetor dos efeitos de repetição, considerados como fixos, somados à média geral; a é o vetor dos efeitos genéticos aditivos individuais (aleatórios), em que a $\sim \mathrm{N}\left(0, \mathrm{~A} \sigma_{\mathrm{a}}^{2}\right)$; $\mathrm{p}$ é o vetor dos efeitos de parcelas (aleatórios), em que $\mathrm{p} \sim \mathrm{N}\left(0, \mathrm{I} \sigma_{\mathrm{p}}^{2}\right)$; e $\varepsilon$ é o vetor de erros (aleatórios), em que $\varepsilon \sim \mathrm{N}\left(0, \mathrm{I} \sigma_{\varepsilon}^{2}\right)$. As letras maiúsculas representam as matrizes de incidência para os referidos efeitos.

A partir destes modelos estatísticos, foram preditos os valores genéticos aditivos das famílias para cada característica, com o auxílio do programa Selegen (Resende, 2007). Para a predição dos valores genéticos, adotou-se o método de modelos lineares mistos via REML/BLUP (máxima verossimilhança restrita/melhor predição linear não enviesada). A partir dos valores genéticos aditivos, obtidos para cada característica, estimou-se a matriz de correlação genética (Resende, 2007).

Para a análise de trilha, realizou-se o diagnóstico de multicolinearidade da matriz de correlação genética. A presença de multicolinearidade foi verificada pela análise dos autovalores da matriz $X^{\prime} X$, em que o grau de multicolinearidade foi estabelecido com base no número de condição (NC), que é a razão entre o maior e o menor autovalor da matriz. Considerou-se também como indicativo de multicolinearidade o fator de inflação da variância (FIV), obtido para cada variável, na diagonal inversa da matriz de correlação ( $\left.X^{\prime} X\right)$.

Depois de constatado moderado grau de multicolinearidade, utilizou-se a análise de regressão em crista ou em cumeeira (Carvalho, 1995), pela qual se adiciona uma constante $(\mathrm{k})$ à diagonal da matriz de correlação $X^{\prime} X$, a fim de reduzir a variância associada ao estimador de mínimos quadrados da análise de trilha. Para selecionar o melhor valor de $\mathrm{k}$ que minimiza a multicolinearidade, verificaram-se as estimativas dos efeitos diretos da análise de trilha (vetor $\widehat{B}^{*}$ ), dos fatores de inflação da variância e do número de condição em função de vários valores de $\mathrm{k}$. Considerou-se como melhor valor de $\mathrm{k}$ a menor constante que possibilitou a estabilização das estimativas do vetor $\widehat{B}^{*}$, FIV inferior a 10 e NC menor que 100 (Hair et al., 2009).

As quatro características de maior interesse para o melhoramento genético de couve são: altura de planta, número de brotações, número de folhas por planta e diâmetro do caule. A seleção nestas características pode possibilitar maior adensamento, rendimento por área $\mathrm{e}$ redução de tratos culturais, assim, foram feitas quatro análises de trilha, em que cada análise considerou uma destas características como variável básica. Para a obtenção do vetor de efeitos diretos, utilizou-se a análise de trilha por meio do método de regressão em crista, pela expressão $\widehat{B} *=\left(R_{x}+k\right)^{-1} R_{x y}$, em que: $\widehat{B} *$ é o vetor com as estimativas dos efeitos diretos; $R_{x}$ é a matriz de correlação entre as variáveis explicativas; $\mathrm{k}$ é o valor da constante $\mathrm{k}$ multiplicada pela matriz identidade, com número de linhas e colunas igual ao número de variáveis explicativas; e $\mathrm{R}_{\mathrm{xy}}$ é o vetor de correlações entre a variável dependente e as variáveis explicativas. 
O diagnóstico de multicolinearidade e análise de trilha foram obtidos a partir de programação no software estatístico R (The R Foundation, 2013).

\section{Resultados e Discussão}

As estimativas de correlações genotípicas obtidas variaram entre -0,680 e 0,901 (Tabela 1). Encontrouse maior estimativa de correlação genotípica entre os caracteres largura e espessura do pecíolo $(0,901)$. Esta estimativa é superior à relatada por Azevedo et al. (2012), que encontrou correlação de 0,380 em 30 acessos de couve. No presente trabalho, também foram encontradas altas estimativas entre as características número de folhas e massa de matéria fresca de folhas $(0,749)$, e entre comprimento e largura de folhas $(0,713)$. Esta estimativa de correlação entre o comprimento e largura de folhas também foi superior à encontrada por Azevedo et al. (2012), que foi de 0,420 . As diferenças entre as estimativas de correlação genotípica encontradas no presente trabalho e as de Azevedo et al. (2012) podem ser explicadas pelas diferenças entre: genótipos avaliados, condições edafoclimáticas e condução do experimento, já que estes autores avaliaram plantas cultivadas em vasos.

Correlações positivas foram encontradas entre o diâmetro do caule e a massa de matéria fresca de folhas $(0,705)$, o que é desejável, pois, além do interesse por plantas produtivas, tem-se interesse por plantas com maior diâmetro de caule, o que reduz a necessidade de tutoramento e diminui a perda de plantas por tombamento (Novo et al., 2010). A correlação negativa
$(-0,671)$ entre o número de brotações e a massa de matéria fresca de folhas também é desejável, pois se consideram melhores as plantas com menor número de brotações e maior produção de massa matéria fresca de folhas. Para as correlações obtidas entre o diâmetro de caule, altura de plantas, número de brotações e número de folhas, as quais foram consideradas como mais importantes no presente trabalho, foram encontradas baixas estimativas (em módulo). Estimativas de correlação genética próximas de zero são indicativas da independência entre as características e, portanto, de que a seleção para uma característica não afeta o desempenho das demais. Porém, para se conhecer as relações de causa e efeito, recomenda-se um estudo mais minucioso das estimativas de correlação por meio da análise de trilha (Teixeira et al., 2012), o que foi feito no presente trabalho.

Segundo Borges et al. (2011), para o estudo correto da análise de trilha, é necessário detectar a presença ou não da multicolinearidade na matriz de correlação entre as variáveis explicativas (matriz X'X). Pelas estimativas do fator de inflação de variância (FIV) e número de condição (NC), verificou-se multicolinearidade severa para a matriz X'X (Tabela 2). As características que mais contribuíram para a multicolinearidade foram: largura do limbo foliar; comprimento do limbo foliar; e razão entre o comprimento e a largura do limbo foliar. A exclusão de uma destas características pode amenizar o problema de multicolinearidade (Toebe \& Cargnelutti Filho, 2013), porém, por serem importantes para o melhoramento da couve, é possível a adição de uma constante $(\mathrm{K})$ na diagonal principal da matriz $\mathrm{X}^{\prime} \mathrm{X}$, antes

Tabela 1. Estimativas dos coeficientes de correlação genotípica, entre características avaliadas em 24 famílias de meios-irmãos de couve.

\begin{tabular}{|c|c|c|c|c|c|c|c|c|c|c|c|c|}
\hline Característica & $\mathrm{NF}$ & MFF & MFF/NF & ALT & $\mathrm{CF}$ & LF & $\mathrm{CF} / \mathrm{LF}$ & $\mathrm{CP}$ & $\mathrm{CF} / \mathrm{CP}$ & LP & EP & $\mathrm{DC}$ \\
\hline$\overline{\mathrm{NB}}$ & $-0,491$ & $-0,671$ & $-0,386$ & 0,103 & $-0,067$ & 0,140 & $-0,302$ & 0,506 & $-0,573$ & 0,111 & 0,051 & $-0,395$ \\
\hline NF & & 0,749 & $-0,067$ & 0,049 & $-0,315$ & $-0,558$ & 0,386 & $-0,381$ & 0,184 & $-0,504$ & $-0,507$ & 0,376 \\
\hline MFF & & & 0,573 & 0,205 & 0,092 & $-0,074$ & 0,262 & $-0,159$ & 0,296 & $-0,185$ & $-0,063$ & 0,705 \\
\hline $\mathrm{MFF} / \mathrm{NF}$ & & & & 0,218 & 0,498 & 0,551 & $-0,081$ & 0,267 & 0,164 & 0,291 & 0,519 & 0,643 \\
\hline ALT & & & & & 0,467 & 0,440 & 0,019 & 0,284 & $-0,018$ & $-0,063$ & 0,064 & 0,117 \\
\hline $\mathrm{CF}$ & & & & & & 0,713 & 0,296 & 0,446 & 0,270 & 0,286 & 0,402 & 0,271 \\
\hline $\mathrm{LF}$ & & & & & & & $-0,453$ & 0,602 & $-0,113$ & 0,530 & 0,625 & 0,196 \\
\hline $\mathrm{CF} / \mathrm{LF}$ & & & & & & & & $-0,248$ & 0,480 & $-0,370$ & $-0,329$ & 0,086 \\
\hline $\mathrm{CP}$ & & & & & & & & & $-0,680$ & 0,384 & 0,346 & 0,033 \\
\hline $\mathrm{CF} / \mathrm{CP}$ & & & & & & & & & & $-0,190$ & $-0,037$ & 0,232 \\
\hline LP & & & & & & & & & & & 0,901 & 0,271 \\
\hline EP & & & & & & & & & & & & 0,380 \\
\hline
\end{tabular}

NB, número de brotações; NF, número de folhas; MFF, massa de folhas frescas; MFF/NF, matéria fresca por folha; ALT, altura da planta; CF, comprimento do limbo foliar; LF, largura do limbo foliar; CF/LF, razão entre o comprimento e a largura do limbo foliar; CP, comprimento do pecíolo; LP, largura do pecíolo; EP, espessura do pecíolo; DC, diâmetro do caule. 
de se realizar a análise de trilha, método denominado de regressão em crista ou cumeeira (Carvalho, 1995).

Em presença de multicolinearidade, as variâncias associadas a certos estimadores, como os coeficientes de trilha, que medem os efeitos diretos de variáveis explicativas sobre uma principal, podem atingir valores demasiadamente elevados (Cruz et al., 2014). Estes valores demasiadamente elevados são evidências de estimativas pouco confiáveis e, consequentemente, sem coerência com o fenômeno biológico estudado, o que foi verificado por Bizeti et al. (2004), Coimbra et al. (2005) e Toebe \& Cargnelutti Filho (2013).

$\mathrm{Na}$ seleção dos melhores valores de $\mathrm{k}$, foram selecionadas as constantes que proporcionaram a estabilização dos efeitos diretos: NC inferior a 100 e FIV inferior a 10 (Figura 1). Segundo Cruz et al. (2014), quanto maior for o valor K, mais enviesadas são as informações obtidas pela análise de trilha.
Por isso, foram selecionados os menores valores de $\mathrm{K}$ que proporcionaram a estabilização dos efeitos diretos - NC inferior a 100 e FIV inferior a 10. Encontramse valores $\mathrm{K}$ de $0,0471,0,0471,0,0449$ e 0,0477 nas análises de trilha, tendo-se considerado como variáveis básicas as características número de brotações, número de folhas, altura de planta e diâmetro do caule, respectivamente. Os valores de $\mathrm{k}$ utilizados no presente trabalho são menores do que os encontrados por Borges et al. (2011) e Toebe \& Cargnelutti Filho (2013), porém foram eficientes para amenizar os problemas de multicolinearidade (Tabela 2). Os menores valores de $\mathrm{k}$ no presente trabalho podem ser consequência do menor grau de multicolinearidade da matriz X'X ou do critério utilizado para a escolha do valor $\mathrm{k}$.

Quando se considerou o número de brotações como variável básica, verificou-se maior efeito direto do comprimento do pecíolo (Tabela 3). A redução

Tabela 2. Multicolinearidade da matriz de correlação genotípica, entre variáveis explicativas ( $\left.\mathrm{X}^{\prime} \mathrm{X}\right)$, em função de valores k de diferentes características como variáveis básicas na avaliação de famílias de meios-irmãos de couve.

\begin{tabular}{|c|c|c|c|c|c|c|c|c|}
\hline \multirow[t]{2}{*}{ Característica } & \multicolumn{2}{|c|}{ NB } & \multicolumn{2}{|c|}{ NF } & \multicolumn{2}{|c|}{ ALT } & \multicolumn{2}{|c|}{$\mathrm{DC}$} \\
\hline & $\mathrm{k}=0,000$ & $\mathrm{k}=0,047$ & $\mathrm{k}=0,000$ & $\mathrm{k}=0,047$ & $\mathrm{k}=0,000$ & $\mathrm{k}=0,0449$ & $\mathrm{k}=0,000$ & $\mathrm{k}=0,0477$ \\
\hline & \multicolumn{8}{|c|}{ Fator de inflação de variância } \\
\hline NB & - & - & 4,723 & 3,049 & 4,527 & 2,961 & 4,445 & 2,923 \\
\hline NF & 21,035 & 6,799 & - & - & 22,911 & 7,136 & 19,241 & 6,168 \\
\hline MFF & 27,337 & 7,574 & 9,159 & 4,694 & 26,301 & 8,023 & 27,331 & 7,712 \\
\hline $\mathrm{MFF} / \mathrm{NF}$ & 14,314 & 4,817 & 5,872 & 3,688 & 16,452 & 5,324 & 14,338 & 4,706 \\
\hline ALT & 2,059 & 1,631 & 2,340 & 1,734 & - & - & 2,257 & 1,733 \\
\hline $\mathrm{CF}$ & 276,692 & 9,186 & 276,815 & 9,178 & 274,690 & 9,299 & 230,780 & 9,054 \\
\hline $\mathrm{LF}$ & 292,483 & 9,967 & 294,956 & 9,968 & 295,535 & 9,967 & 256,511 & 9,976 \\
\hline $\mathrm{CF} / \mathrm{LF}$ & 150,316 & 5,404 & 151,449 & 5,398 & 151,481 & 5,634 & 129,666 & 5,352 \\
\hline $\mathrm{CP}$ & 11,076 & 5,106 & 14,247 & 6,024 & 13,138 & 6,018 & 13,382 & 5,975 \\
\hline $\mathrm{CF} / \mathrm{CP}$ & 11,997 & 4,699 & 12,015 & 4,672 & 11,239 & 4,708 & 11,778 & 4,647 \\
\hline LP & 12,727 & 4,998 & 12,977 & 5,200 & 14,536 & 5,321 & 14,767 & 5,049 \\
\hline EP & 16,597 & 5,924 & 17,335 & 6,014 & 17,463 & 6,102 & 17,386 & 5,925 \\
\hline DC & 4,706 & 2,913 & 4,575 & 2,768 & 5,255 & 3,129 & - & - \\
\hline$\overline{\lambda_{j}}$ & \multicolumn{8}{|c|}{ Autovalores da matriz de correlação (X'X) } \\
\hline$\lambda_{1}$ & 4,186 & 4,233 & 3,923 & 3,970 & 4,222 & 4,267 & 4,270 & 4,318 \\
\hline$\lambda_{2}$ & 3,000 & 3,047 & 3,237 & 3,284 & 3,438 & 3,482 & 2,934 & 2,982 \\
\hline$\lambda_{3}$ & 1,507 & 1,554 & 1,568 & 1,615 & 1,447 & 1,492 & 1,553 & 1,601 \\
\hline$\lambda_{4}$ & 1,423 & 1,470 & 1,206 & 1,253 & 1,228 & 1,273 & 1,332 & 1,380 \\
\hline$\lambda_{5}$ & 0,784 & 0,831 & 0,792 & 0,839 & 0,725 & 0,770 & 0,770 & 0,817 \\
\hline$\lambda_{6}$ & 0,542 & 0,589 & 0,528 & 0,575 & 0,384 & 0,429 & 0,541 & 0,588 \\
\hline$\lambda_{7}$ & 0,255 & 0,302 & 0,354 & 0,402 & 0,307 & 0,352 & 0,295 & 0,343 \\
\hline$\lambda_{8}$ & 0,182 & 0,229 & 0,223 & 0,270 & 0,134 & 0,178 & 0,197 & 0,245 \\
\hline$\lambda_{9}$ & 0,058 & 0,105 & 0,089 & 0,137 & 0,055 & 0,100 & 0,050 & 0,098 \\
\hline$\lambda_{10}$ & 0,046 & 0,093 & 0,041 & 0,088 & 0,042 & 0,087 & 0,038 & 0,086 \\
\hline$\lambda_{11}$ & 0,017 & 0,064 & 0,036 & 0,083 & 0,016 & 0,061 & 0,018 & 0,066 \\
\hline$\lambda_{12}$ & 0,001 & 0,048 & 0,001 & 0,048 & 0,001 & 0,046 & 0,002 & 0,049 \\
\hline Número de condição & 3048,994 & 87,333 & 2867,171 & 81,910 & 3088,205 & 92,230 & 2674,788 & 87,585 \\
\hline
\end{tabular}

NB, número de brotações; NF, número de folhas; MFF, massa de folhas frescas; MFF/NF, matéria fresca por folha; ALT, altura da planta; CF, comprimento do limbo foliar; LF, largura do limbo foliar; CF/LF, razão entre o comprimento e a largura do limbo foliar; CP, comprimento do pecíolo; LP, largura do pecíolo; EP, espessura do pecíolo; DC, diâmetro do caule. 


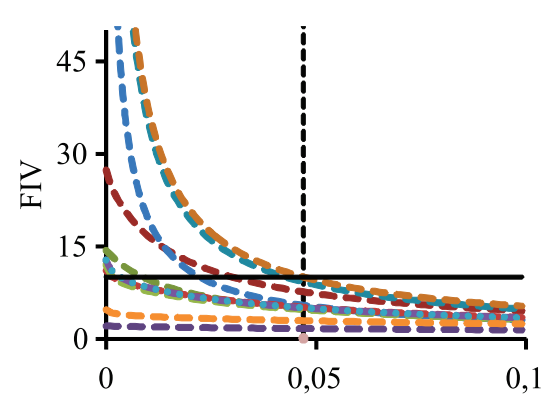

Variável básica (número de brotações)
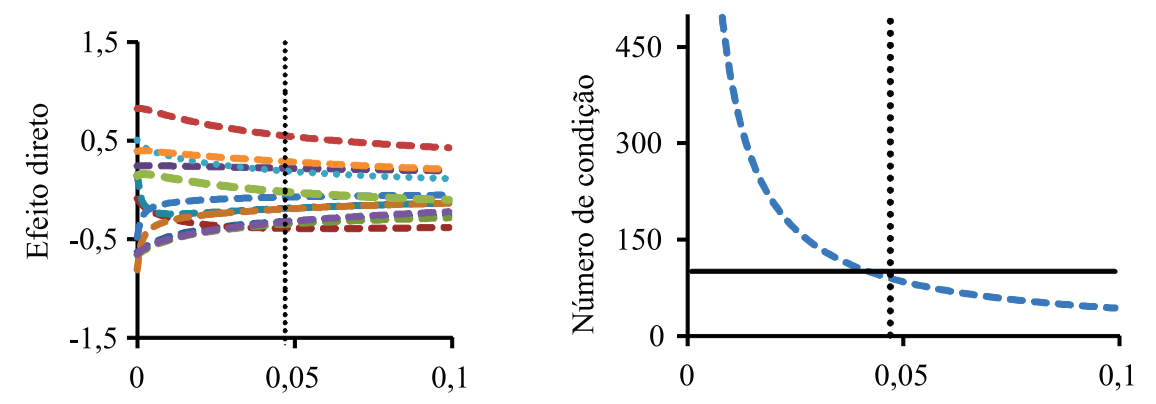

Variável básica (número de folhas)
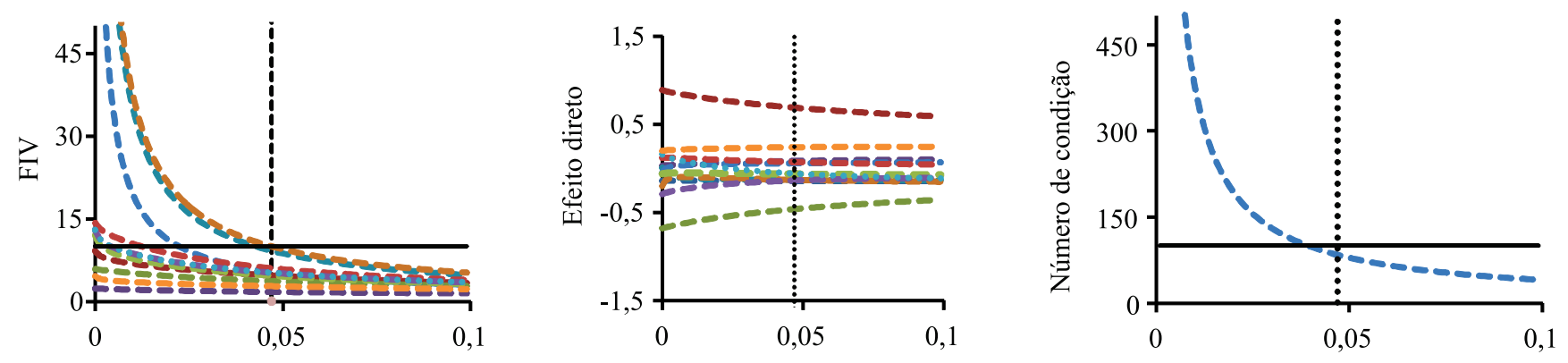

Variável básica (altura da planta)
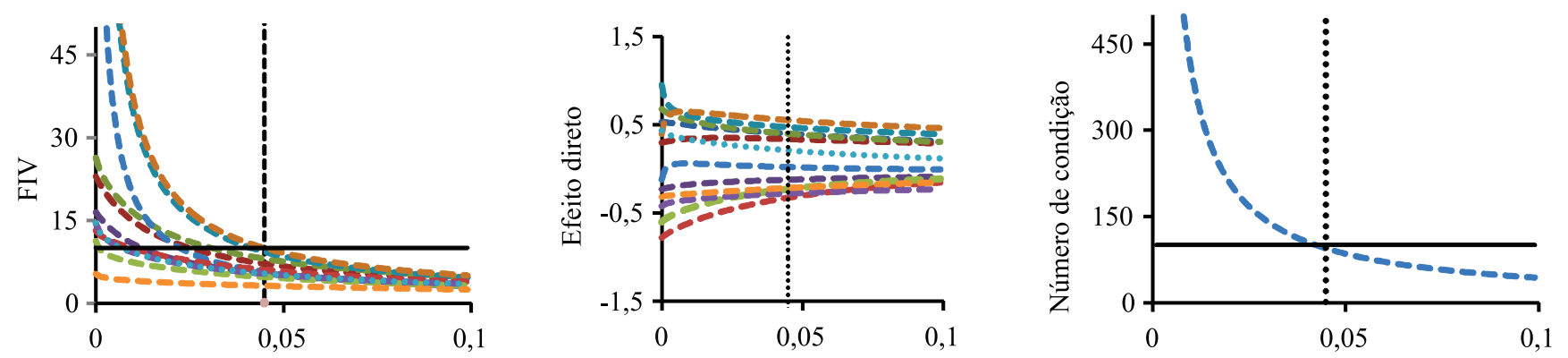

Variável básica (diâmetro do caule)
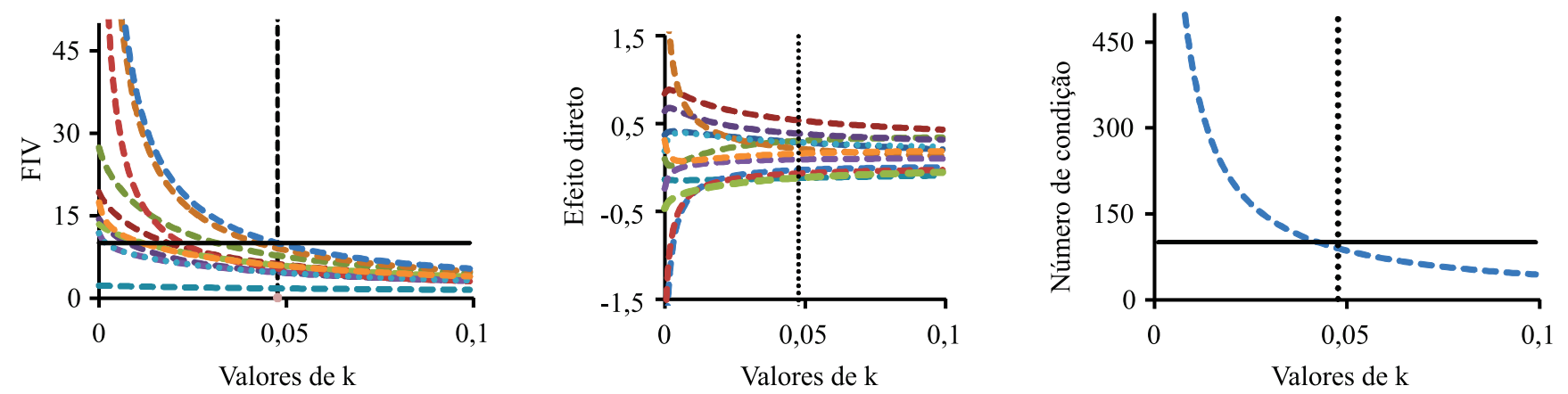

Figura 1. Estimativas dos fatores de inflação da variância (FIV), número de condição e efeitos diretos da análise de trilha, em função de valores de K, no estudo da multicolinearidade em famílias de meios-irmãos de couve. 
do número de brotações é desejável, pois facilita os tratos culturais, diminuindo a necessidade de desbrota. Quanto ao comprimento do pecíolo, são desejáveis plantas com comprimento próximo de $10 \mathrm{~cm}$, por facilitar a amarração, o que geralmente é feito para a comercialização in natura. Encontrou-se estimativa negativa do efeito direto da massa de matéria fresca de folhas sobre o número de brotações, o que é desejável, pois há interesse em reduzir o número de brotações e aumentar a massa de matéria fresca de folhas. Porém, este efeito foi menor do que o da variável residual, o que mostra que estas características não são fortemente associadas.

Ao se considerar o número de folhas como variável básica, encontraram-se efeitos diretos, que superaram o efeito da variável residual, para as características massa de matéria fresca de folha e massa de matéria fresca por folha (Tabela 3). Estas associações

Tabela 3. Estimativas dos efeitos diretos e indiretos da análise de trilha com regressão em crista, no estudo de meios-irmãos de couve, em que se consideram o número de brotações e o número de folhas como variáveis básicas.

\begin{tabular}{|c|c|c|c|c|c|c|c|c|c|c|c|c|}
\hline \multirow[t]{2}{*}{ EI Via } & \multicolumn{12}{|c|}{ Variáveis explicativas } \\
\hline & $\mathrm{NF}$ ou $\mathrm{NB}^{(1)}$ & MFF & $\mathrm{MFF} / \mathrm{NF}$ & ALT & $\mathrm{CF}$ & LF & $\mathrm{CF} / \mathrm{LF}$ & $\mathrm{CP}$ & $\mathrm{CF} / \mathrm{CP}$ & LP & EP & $\mathrm{DC}$ \\
\hline & \multicolumn{12}{|c|}{ Variável básica: número de brotações } \\
\hline NF & - & $-0,240$ & 0,021 & $-0,016$ & 0,101 & 0,179 & $-0,123$ & 0,122 & $-0,059$ & 0,161 & 0,162 & $-0,120$ \\
\hline MFF & $-0,293$ & - & $-0,224$ & $-0,080$ & $-0,036$ & 0,029 & $-0,103$ & 0,062 & $-0,116$ & 0,072 & 0,025 & $-0,276$ \\
\hline $\mathrm{MFF} / \mathrm{NF}$ & 0,023 & $-0,200$ & - & $-0,076$ & $-0,174$ & $-0,193$ & 0,028 & $-0,093$ & $-0,057$ & $-0,102$ & $-0,182$ & $-0,225$ \\
\hline ALT & 0,011 & 0,045 & 0,048 & - & 0,102 & 0,096 & 0,004 & 0,062 & $-0,004$ & $-0,014$ & 0,014 & 0,025 \\
\hline $\mathrm{CF}$ & 0,059 & $-0,017$ & $-0,094$ & $-0,088$ & - & $-0,134$ & $-0,056$ & $-0,084$ & $-0,051$ & $-0,054$ & $-0,076$ & $-0,051$ \\
\hline LF & 0,109 & 0,014 & $-0,107$ & $-0,086$ & $-0,139$ & - & 0,088 & $-0,117$ & 0,022 & $-0,103$ & $-0,122$ & $-0,038$ \\
\hline $\mathrm{CF} / \mathrm{LF}$ & $-0,029$ & $-0,019$ & 0,006 & $-0,001$ & $-0,022$ & 0,034 & - & 0,018 & $-0,036$ & 0,027 & 0,024 & $-0,006$ \\
\hline $\mathrm{CP}$ & $-0,208$ & $-0,087$ & 0,146 & 0,155 & 0,244 & 0,329 & $-0,135$ & - & $-0,372$ & 0,210 & 0,189 & 0,018 \\
\hline $\mathrm{CF} / \mathrm{CP}$ & $-0,004$ & $-0,006$ & $-0,003$ & 0,000 & $-0,006$ & 0,002 & $-0,010$ & 0,014 & - & 0,004 & 0,001 & $-0,005$ \\
\hline LP & 0,164 & 0,060 & $-0,094$ & 0,020 & $-0,093$ & $-0,172$ & 0,120 & $-0,125$ & 0,062 & - & $-0,292$ & $-0,088$ \\
\hline EP & $-0,096$ & $-0,012$ & 0,099 & 0,012 & 0,076 & 0,119 & $-0,062$ & 0,066 & $-0,007$ & 0,171 & - & 0,072 \\
\hline DC & 0,107 & 0,202 & 0,184 & 0,033 & 0,078 & 0,056 & 0,025 & 0,009 & 0,066 & 0,077 & 0,109 & - \\
\hline ED sobre NB & $-0,320$ & $-0,391$ & $-0,350$ & 0,218 & $-0,188$ & $-0,195$ & $-0,074$ & 0,546 & $-0,021$ & $-0,325$ & 0,190 & 0,286 \\
\hline Correlação total & $-0,491$ & $-0,671$ & $-0,386$ & 0,103 & $-0,067$ & 0,140 & $-0,302$ & 0,506 & $-0,573$ & 0,111 & 0,051 & $-0,395$ \\
\hline$\overline{\mathrm{R}^{2}}$ & \multicolumn{12}{|c|}{0,734} \\
\hline $\mathrm{k}$ & \multicolumn{12}{|c|}{0,047} \\
\hline \multirow[t]{2}{*}{$\underline{\mathrm{Pe}}$} & \multicolumn{12}{|c|}{0,516} \\
\hline & \multicolumn{12}{|c|}{ Variável básica: número de folhas } \\
\hline NB & - & 0,096 & 0,055 & $-0,015$ & 0,010 & $-0,020$ & 0,043 & $-0,073$ & 0,082 & $-0,016$ & $-0,007$ & 0,057 \\
\hline MFF & $-0,461$ & - & 0,394 & 0,141 & 0,064 & $-0,051$ & 0,180 & $-0,109$ & 0,203 & $-0,127$ & $-0,043$ & 0,485 \\
\hline $\mathrm{MFF} / \mathrm{NF}$ & 0,178 & $-0,265$ & - & $-0,101$ & $-0,230$ & $-0,255$ & 0,037 & $-0,123$ & $-0,076$ & $-0,134$ & $-0,240$ & $-0,297$ \\
\hline ALT & 0,009 & 0,017 & 0,018 & - & 0,039 & 0,037 & 0,002 & 0,024 & $-0,002$ & $-0,005$ & 0,005 & 0,010 \\
\hline $\mathrm{CF}$ & 0,009 & $-0,012$ & $-0,065$ & $-0,061$ & - & $-0,093$ & $-0,039$ & $-0,058$ & $-0,035$ & $-0,037$ & $-0,052$ & $-0,035$ \\
\hline LF & $-0,018$ & 0,010 & $-0,072$ & $-0,057$ & $-0,093$ & - & 0,059 & $-0,078$ & 0,015 & $-0,069$ & $-0,081$ & $-0,025$ \\
\hline $\mathrm{CF} / \mathrm{LF}$ & $-0,017$ & 0,015 & $-0,005$ & 0,001 & 0,017 & $-0,026$ & - & $-0,014$ & 0,028 & $-0,021$ & $-0,019$ & 0,005 \\
\hline $\mathrm{CP}$ & 0,036 & $-0,011$ & 0,019 & 0,020 & 0,032 & 0,043 & $-0,018$ & - & $-0,048$ & 0,027 & 0,025 & 0,002 \\
\hline $\mathrm{CF} / \mathrm{CP}$ & 0,037 & $-0,019$ & $-0,011$ & 0,001 & $-0,017$ & 0,007 & $-0,031$ & 0,044 & - & 0,012 & 0,002 & $-0,015$ \\
\hline LP & $-0,015$ & 0,025 & $-0,040$ & 0,009 & $-0,039$ & $-0,073$ & 0,051 & $-0,053$ & 0,026 & - & $-0,124$ & $-0,037$ \\
\hline EP & $-0,003$ & 0,004 & $-0,031$ & $-0,004$ & $-0,024$ & $-0,038$ & 0,020 & $-0,021$ & 0,002 & $-0,054$ & - & $-0,023$ \\
\hline DC & $-0,094$ & 0,168 & 0,154 & 0,028 & 0,065 & 0,047 & 0,021 & 0,008 & 0,055 & 0,065 & 0,091 & - \\
\hline ED sobre NF & $-0,144$ & 0,688 & $-0,462$ & 0,084 & $-0,131$ & $-0,130$ & 0,057 & 0,071 & $-0,064$ & $-0,138$ & $-0,060$ & 0,239 \\
\hline Correlação total & $-0,491$ & 0,749 & $-0,067$ & 0,049 & $-0,315$ & $-0,558$ & 0,386 & $-0,381$ & 0,184 & $-0,504$ & $-0,507$ & 0,376 \\
\hline$\overline{\mathrm{R}^{2}}$ & \multicolumn{12}{|c|}{0,907} \\
\hline $\mathrm{k}$ & \multicolumn{12}{|c|}{0,047} \\
\hline$\underline{\mathrm{P}_{\mathrm{e}}}$ & \multicolumn{12}{|c|}{0,305} \\
\hline
\end{tabular}


apresentaram resultado desejado para o melhoramento da couve, pois, além de se esperar um maior número de folhas comercializáveis, é necessário também que se obtenha maior produção de massa de matéria fresca de folha por planta e redução da massa de matéria fresca por folha. A redução da massa de matéria fresca por folha é desejável nesta população, já que a maioria das plantas avaliadas tem folhas de grande dimensão, geralmente mais espessas e duras, o que é de menor interesse comercial. Embora a massa de matéria fresca por folha tenha sido a segunda característica com maior efeito direto sobre o número de folhas, a estimativa de correlação genética entre estas características foi baixa $(-0,067)$. Estimativas dos efeitos diretos superiores à estimativa de correlação mostram que as conclusões baseadas em correlações podem induzir a erros (Teixeira et al., 2012); neste caso, indica-se o uso da análise de trilha.

Tabela 4. Estimativas dos efeitos diretos e indiretos da análise de trilha com regressão em crista, no estudo de meios-irmãos de couve, considerando-se a altura da planta e o diâmetro do caule como variáveis básicas.

\begin{tabular}{|c|c|c|c|c|c|c|c|c|c|c|c|c|}
\hline \multirow[t]{2}{*}{$\overline{\text { EI Via }}$} & \multicolumn{12}{|c|}{ Variáveis explicativas } \\
\hline & NB & NF & MFF & $\mathrm{MFF} / \mathrm{NF}$ & $\mathrm{CF}$ & LF & $\mathrm{CF} / \mathrm{LF}$ & $\mathrm{CP}$ & $\mathrm{CF} / \mathrm{CP}$ & LP & EP & $\mathrm{DC}_{\text {ou }} \mathrm{ALT}^{(1)}$ \\
\hline & \multicolumn{12}{|c|}{ Variável básica: altura de planta } \\
\hline NB & - & $-0,194$ & $-0,265$ & $-0,153$ & $-0,026$ & 0,055 & $-0,120$ & 0,200 & $-0,227$ & 0,044 & 0,020 & $-0,156$ \\
\hline NF & $-0,165$ & - & 0,251 & $-0,022$ & $-0,106$ & $-0,187$ & 0,129 & $-0,128$ & 0,062 & $-0,169$ & $-0,170$ & 0,126 \\
\hline MFF & $-0,266$ & 0,297 & - & 0,227 & 0,037 & $-0,029$ & 0,104 & $-0,063$ & 0,117 & $-0,073$ & $-0,025$ & 0,280 \\
\hline $\mathrm{MFF} / \mathrm{NF}$ & 0,048 & 0,008 & $-0,071$ & - & $-0,062$ & $-0,069$ & 0,010 & $-0,033$ & $-0,020$ & $-0,036$ & $-0,065$ & $-0,080$ \\
\hline $\mathrm{CF}$ & $-0,031$ & $-0,148$ & 0,043 & 0,234 & - & 0,335 & 0,139 & 0,210 & 0,127 & 0,134 & 0,189 & 0,128 \\
\hline $\mathrm{LF}$ & 0,077 & $-0,307$ & $-0,041$ & 0,303 & 0,393 & - & $-0,249$ & 0,332 & $-0,062$ & 0,292 & 0,344 & 0,108 \\
\hline $\mathrm{CF} / \mathrm{LF}$ & $-0,005$ & 0,007 & 0,005 & $-0,001$ & 0,005 & $-0,008$ & - & $-0,004$ & 0,009 & $-0,007$ & $-0,006$ & 0,002 \\
\hline $\mathrm{CP}$ & $-0,166$ & 0,125 & 0,052 & $-0,088$ & $-0,146$ & $-0,198$ & 0,081 & - & 0,223 & $-0,126$ & $-0,114$ & $-0,011$ \\
\hline $\mathrm{CF} / \mathrm{CP}$ & 0,132 & $-0,042$ & $-0,068$ & $-0,038$ & $-0,062$ & 0,026 & $-0,111$ & 0,157 & - & 0,044 & 0,008 & $-0,054$ \\
\hline LP & $-0,031$ & 0,141 & 0,052 & $-0,082$ & $-0,080$ & $-0,148$ & 0,104 & $-0,108$ & 0,053 & - & $-0,252$ & $-0,076$ \\
\hline EP & 0,011 & $-0,105$ & $-0,013$ & 0,108 & 0,083 & 0,130 & $-0,068$ & 0,072 & $-0,008$ & 0,187 & - & 0,079 \\
\hline $\mathrm{DC}$ & 0,086 & $-0,082$ & $-0,154$ & $-0,141$ & $-0,059$ & $-0,043$ & $-0,019$ & $-0,007$ & $-0,051$ & $-0,059$ & $-0,083$ & - \\
\hline ED sobre ALT & 0,396 & 0,336 & 0,396 & $-0,124$ & 0,47 & 0,551 & 0,018 & $-0,328$ & $-0,231$ & $-0,280$ & 0,207 & $-0,219$ \\
\hline Corr. total & 0,103 & 0,049 & 0,205 & 0,218 & 0,467 & 0,44 & 0,019 & 0,284 & $-0,018$ & $-0,063$ & 0,064 & 0,117 \\
\hline$\overline{\mathrm{R}^{2}}$ & & & & & & & 489 & & & & & \\
\hline $\mathrm{k}$ & & & & & & & 045 & & & & & \\
\hline \multirow[t]{2}{*}{$\mathrm{Pe}$} & & & & & & & 715 & & & & & \\
\hline & \multicolumn{12}{|c|}{ Variável básica: diâmetro do caule } \\
\hline NB & - & $-0,141$ & $-0,192$ & $-0,111$ & $-0,019$ & 0,040 & $-0,087$ & 0,145 & $-0,164$ & 0,032 & 0,015 & 0,029 \\
\hline NF & $-0,262$ & - & 0,400 & $-0,036$ & $-0,168$ & $-0,298$ & 0,206 & $-0,203$ & 0,098 & $-0,269$ & $-0,271$ & 0,026 \\
\hline MFF & $-0,202$ & 0,225 & - & 0,172 & 0,028 & $-0,022$ & 0,079 & $-0,048$ & 0,089 & $-0,056$ & $-0,019$ & 0,062 \\
\hline $\mathrm{MFF} / \mathrm{NF}$ & $-0,148$ & $-0,026$ & 0,220 & - & 0,191 & 0,211 & $-0,031$ & 0,102 & 0,063 & 0,112 & 0,199 & 0,084 \\
\hline $\mathrm{CF}$ & $-0,014$ & $-0,067$ & 0,020 & 0,106 & - & 0,152 & 0,063 & 0,095 & 0,057 & 0,061 & 0,085 & 0,099 \\
\hline LF & $-0,004$ & 0,017 & 0,002 & $-0,016$ & $-0,021$ & - & 0,014 & $-0,018$ & 0,003 & $-0,016$ & $-0,019$ & $-0,013$ \\
\hline $\mathrm{CF} / \mathrm{LF}$ & 0,021 & $-0,027$ & $-0,018$ & 0,006 & $-0,020$ & 0,031 & - & 0,017 & $-0,033$ & 0,025 & 0,023 & $-0,001$ \\
\hline $\mathrm{CP}$ & $-0,060$ & 0,045 & 0,019 & $-0,032$ & $-0,053$ & $-0,072$ & 0,030 & - & 0,081 & $-0,046$ & $-0,041$ & $-0,034$ \\
\hline $\mathrm{CF} / \mathrm{CP}$ & $-0,052$ & 0,017 & 0,027 & 0,015 & 0,024 & $-0,010$ & 0,044 & $-0,062$ & - & $-0,017$ & $-0,003$ & $-0,002$ \\
\hline LP & 0,031 & $-0,142$ & $-0,052$ & 0,082 & 0,081 & 0,150 & $-0,105$ & 0,109 & $-0,054$ & - & 0,255 & $-0,018$ \\
\hline EP & 0,008 & $-0,079$ & $-0,010$ & 0,081 & 0,063 & 0,098 & $-0,052$ & 0,054 & $-0,006$ & 0,141 & - & 0,010 \\
\hline ALT & $-0,012$ & $-0,006$ & $-0,025$ & $-0,026$ & $-0,056$ & $-0,053$ & $-0,002$ & $-0,034$ & 0,002 & 0,008 & $-0,008$ & - \\
\hline ED sobre DC & 0,287 & 0,534 & 0,301 & 0,383 & 0,213 & $-0,030$ & $-0,069$ & $-0,119$ & 0,091 & 0,283 & 0,157 & $-0,121$ \\
\hline Corr. total & $-0,395$ & 0,376 & 0,705 & 0,643 & 0,271 & 0,196 & 0,086 & 0,033 & 0,232 & 0,271 & 0,380 & 0,117 \\
\hline$\overline{\mathrm{R}^{2}}$ & \multicolumn{12}{|c|}{0,732} \\
\hline $\mathrm{k}$ & \multicolumn{12}{|c|}{0,048} \\
\hline$\underline{\mathrm{P}_{\mathrm{e}}}$ & \multicolumn{12}{|c|}{0,518} \\
\hline
\end{tabular}

Pesq. agropec. bras., Brasília, v.51, n.1, p.35-44, jan. 2016

DOI: 10.1590/S0100-204X2016000100005 
$\mathrm{Na}$ altura da planta, não se encontraram estimativas de efeitos diretos e indiretos superiores ao efeito da variável residual (Tabela 4), o que indica que a seleção truncada quanto à altura de plantas pode não trazer consequências para as demais características. A média para a altura de plantas, no presente experimento, foi de $87 \mathrm{~cm}$, maior que o desejável, pois, de acordo com Novo et al. (2010), a maioria das cultivares comercializadas no Brasil tem porte que varia de 40 a $80 \mathrm{~cm}$, as quais são multiplicadas por brotações. As cultivares híbridas são ainda mais compactas, com altura de até $50 \mathrm{~cm}$ e multiplicadas por sementes. A obtenção de plantas de couve de menor altura é vantajosa, pois possibilita maior adensamento, o que proporciona maior produção de folhas por área (Naik \& Gupta, 2010). Quando se considerou o diâmetro de caule como variável básica, verificou-se maior efeito direto da característica massa de matéria fresca de folha. A associação positiva entre estas características é desejável, pois há o interesse em
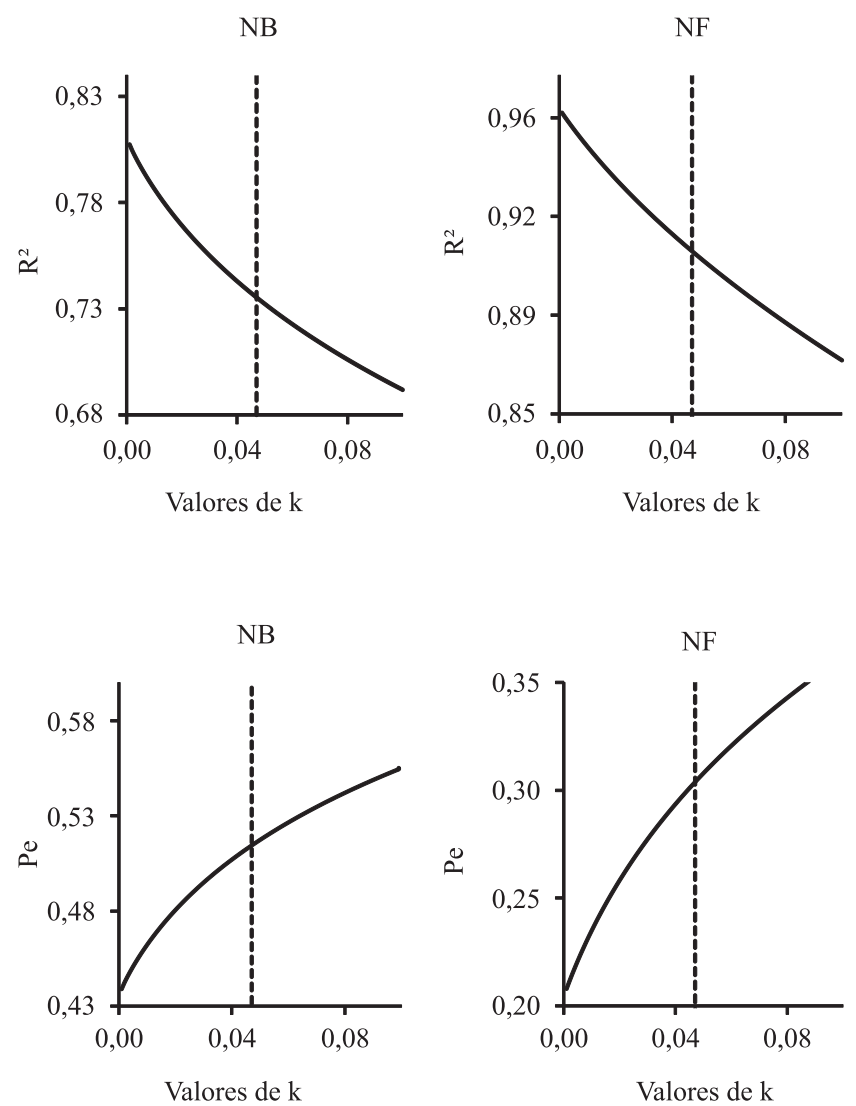

aumentar o diâmetro do caule e a massa de matéria fresca de folha (Novo et al., 2010).

Em geral, encontraram-se coeficientes de determinação satisfatórios para as análises de trilha, com estimativas superiores a 0,700 , com exceção da análise que considerou a altura de plantas como variável básica $(0,489)$. Este baixo coeficiente de determinação $(0,489)$ é uma consequência das baixas estimativas de correlação da variável básica (altura de plantas) com todas as variáveis explicativas (Tabela 1). Nesta situação, o modelo não contempla todas as variáveis necessárias para explicar a variável básica e, consequentemente, o coeficiente de determinação é baixo (Cruz et al., 2012). Outra justificativa, quando se realiza a análise de trilha com regressão em cumeeira, é a soma da constante $\mathrm{k}$ na diagonal da matriz $\mathrm{X}^{\prime} \mathrm{X}$, que tende a reduzir o coeficiente de determinação $e$ a aumentar o valor do efeito da variável residual na análise de trilha (Figura 2).
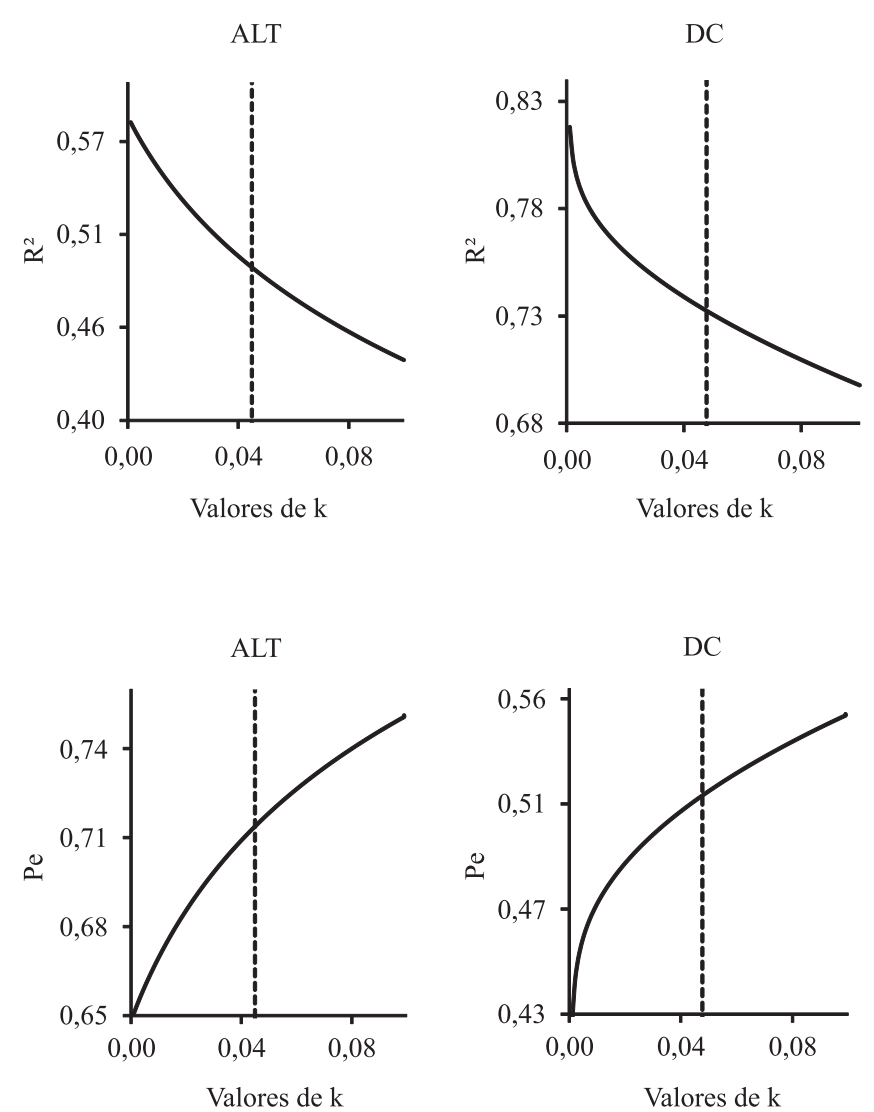

Figura 2. Estimativas do coeficiente de determinação $\left(\mathrm{R}^{2}\right)$ e do efeito da variável residual $(\mathrm{Pe})$ para a análise de trilha, em função de valores de $\mathrm{K}$, com as variáveis básicas número de brotações (NB), número de folhas (NF), altura de plantas (ALT) e diâmetro do caule (DC), avaliadas em famílias de meios-irmãos de couve. 


\section{Conclusões}

1. A seleção quanto ao menor número de brotações ocasiona a redução do comprimento do pecíolo.

2. A seleção quanto ao maior número de folhas proporciona o aumento da produção de massa de matéria fresca de folhas e a redução da massa de matéria fresca por folha.

3. A seleção quanto ao maior diâmetro do caule ocasiona o aumento da produção de massa de matéria fresca de folhas.

4. A seleção truncada quanto à altura de plantas não ocasiona alterações expressivas nas demais características avaliadas.

\section{Agradecimentos}

Ao Conselho Nacional de Desenvolvimento Científico e Tecnológico (CNPq), à Fundação de Amparo à Pesquisa do Estado de Minas Gerais (Fapemig) e à Coordenação de Aperfeiçoamento de Pessoal de Nível Superior (Capes), pela concessão de bolsas e pelo apoio financeiro.

\section{Referências}

AZEVEDO, A.M.; ANDRADE JÚNIOR, V.C. de; PEDROSA, C.E.; FERNANDES, J.S.C.; VALADARES, N.R.; FERREIRA, M.R.A.; MARTINS, R.A. do V. Desempenho agronômico e variabilidade genética em genótipos de couve. Pesquisa Agropecuária Brasileira, v.47, p.1751-1758, 2012. DOI: 10.1590/S0100-204X2012001200011.

AZEVEDO, A.M.; ANDRADE JÚNIOR, V.C.; FERNANDES, J.S.C; PEDROSA, C.E.; VALADARES, N.R.; FERREIRA, M.R.M.; MARTINS, R.A.V. Divergência genética e importância de caracteres morfológicos em genótipos de couve. Horticultura Brasileira, v.32, p.48-54, 2014. DOI: 10.1590/S0102-05362014000100008.

BALKAYA, A.; YANMAZ, R. Promising kale (Brassica oleracea var. acephala) populations from Black Sea region, Turkey. New Zealand Journal of Crop and Horticultural Science, v.33, p.1-7, 2005. DOI: 10.1080/01140671.2005.9514324.

BIZETI, H.S.; CARVALHO, C.G.P. de; SOUZA, J.R.P. de; DESTRO, D. Path analysis under multicollinearity in soybean. Brazilian Archives of Biology and Technology, v.47, p.669-676, 2004. DOI: 10.1590/S1516-89132004000500001.

BORGES, V.; SOUZA SOBRINHO, F.; LÉDO, F.J. da S.; KOPP, M.M. Associação entre caracteres e análise de trilha na seleção de progênies de meios-irmãos de Brachiaria ruziziensis. Revista Ceres, v.58, p.765-772, 2011. DOI: 10.1590/S0034-737X2011000600013.

CARVALHO, S.P. de. Métodos alternativos de estimação de coeficientes de trilha e índices de seleção sob multicolinearidade.
1994. 163p. Tese (Doutorado) - Universidade Federal de Viçosa, Viçosa, 1995.

COIMBRA, J.L.M.; BENIN, G.; VIEIRA, E.A.; OLIVEIRA, A.C. de; CARVALHO, F.I.F.; GUIDOLIN, A.F.; SOARES, A.P. Consequências da multicolinearidade sobre a análise de trilha em canola. Ciência Rural, v.35, p.347-352, 2005. DOI: 10.1590/ S0103-84782005000200015.

CRUZ, C.D.; CARNEIRO, P.C.S.; REGAZZI, A.J. Modelos biométricos aplicados ao melhoramento genético. 3.ed. rev. ampl. Viçosa: Ed. UFV, 2014. v.2, 668p.

CRUZ, C.D.; REGAZZI, A.J.; CARNEIRO, P.C.S. Modelos biométricos aplicados ao melhoramento genético. 4.ed. Viçosa: Ed. UFV, 2012. v.1, 514p.

ENTRINGER, G.C.; SANTOS, P.H.A.D.; VETTORAZZI, J.C.F; CUNHA, K.S. da; PEREIRA, M.G. Correlação e análise de trilha para componentes de produção de milho superdoce. Revista Ceres, v.61, p.356-361, 2014. DOI: 10.1590/S0034-737X2014000300009.

HAIR, J.F.; BLACK, W.C.; BABIN, B.J.; ANDERSON, R.E.; TATHAM, R.L. Análise multivariada de dados. 6.ed. Porto Alegre: Bookman, 2009. 688p.

MORENO, D.A.; CARVAJAL, M.; LOPEZ-BERENGUER, C.; GARCIA-VIGUERA, C. Chemical and biological characterization of nutraceutical compounds of broccoli. Journal of Pharmaceutical and Biomedical Analysis, v.41, p.1508-1522, 2006. DOI: 10.1016/j. jpba.2006.04.003.

NAIK, I.A.; GUPTA, A.J. Effect of plant density and integrated nutrient management on growth, yield, quality and economics of kale (Brassica oleracea var. acephala) in temperate region. Indian Journal of Agricultural Sciences, v.80, p.80-84, 2010.

NOVO, M. do C. de S.S.; PRELA-PANTANO, A.; TRANI, P.E.; BLAT, S.F. Desenvolvimento e produção de genótipos de couve manteiga. Horticultura Brasileira, v.28, p.321-325, 2010. DOI: 10.1590/S0102-05362010000300014.

RESENDE, M.D.V. de. Matemática e estatística na análise de experimentos e no melhoramento genético. Colombo: Embrapa Florestas, 2007. 561p.

SOENGAS, P.; SOTElO, T.; VELASCO, P.; CARTEA M.E. Antioxidant properties of Brassica vegetables. Functional Plant Science and Biotechnology, v.5, p.43-55, 2011.

TEIXEIRA, D.H.L.; OLIVEIRA, M. do S.P. de; GONÇALVES, F.M.A.; NUNES, J.A.R. Correlações genéticas e análise de trilha para componentes da produção de frutos de açaizeiro. Revista Brasileira de Fruticultura, v.34, p.1135-1142, 2012. DOI: 10.1590/ S0100-29452012000400022.

THE R FOUNDATION. R: the R project for statistical computing. R Vienna: The R Foundation, 2013.

TOEBE, M.; CARGNELUTTI FILHO, A. Não normalidade multivariada e multicolinearidade na análise de trilha em milho. Pesquisa Agropecuária Brasileira, v.48, p.466-477, 2013. DOI: 10.1590/S0100-204X2013000500002.

VILAR, M.; CARTEA, M.E.; PADILLA, G. The potential of kales as a promising vegetable crop. Euphytica, v.159, v.153-165, 2008.

Recebido em 6 de julho de 2015 e aprovado em 6 de outubro de 2015 